\title{
A Novel Edge Detector for Color Images Based on MCDM with Evidential Reasoning
}

\author{
Ruhuan Li, Deqiang Han \\ Inst. of Integrated Automation \\ Xian Jiaotong University \\ Xian, Shaanxi, China 710049 \\ xjtulrh@stu.xjtu.edu.cn; \\ deqhan@gmail.com
}

\author{
Jean Dezert \\ ONERA \\ The French Aerospace Lab \\ Chemin de la Hunière \\ F-91761 Palaiseau, France \\ jean.dezert@onera.fr
}

\author{
Yi Yang \\ SKLSVMS \\ School of Aerospace \\ Xian Jiaotong University \\ Xian, Shaanxi, China 710049 \\ jiafeiyy@mail.xjtu.edu.cn
}

\begin{abstract}
Edge detection is one of the most important tasks in image processing and pattern recognition. Edge detector with multiple color channels can provide more edge information. However, the uncertainty occurring with the edge detection in each single channel and the discordance existing in the fusion of multiple channels edge detectors make the detection difficult. In this paper, we propose a new edge detection method in color images based on information fusion. We show that obtaining final edge through fusing the edge information in each channel is a challenging problem to make decision in the framework of Multi-Criteria decision making (MCDM). In this work, we propose to detect edges in color images using Cautious OWA with evidential reasoning (COWA-ER) and Fuzzy-Cautious OWA with evidential reasoning (FCOWA-ER) to handle the uncertainty and discordance. Experimental results show that the proposed approaches achieve better edge detection performance compared with the original edge detector.
\end{abstract}

Index Terms-Edge detection, uncertainty, Evidence theory, COWA-ER, FCOWA-ER, information fusion.

\section{INTRODUCTION}

Since the edge is one of the main features of the image, edge detection is one of the most important foundations of digital image processing, pattern recognition and computer vision. Edge detection technologies have been widely used in image segmentation [1], motion detection [2] and face recognition [3], etc. According to the type of image, edge detection can be divided into two categories including gray image edge detection and color image edge detection [4].

Classical gray image edge detection methods include first order differential edge detectors (e.g., Roberts [5], Prewitt [6], Sobel [7], Kirsch [8]), second order differential edge detectors (e.g., Laplacian operator and LOG operator), and other detectors, (e.g., Canny [9] and SUSAN [10]).

Classical color image edge detection methods can be divided into scalar methods and vector methods [11], [12]. Scalar methods include the gray conversion based method and multichannel information fusion method. The gray conversion method first converts the color image into a gray image, then uses the gray image edge detection method to detect the edge. It is simple, but a lot of chroma information could be lost during the conversion. The channels fusion method first treats information from the individual color channels separately, then combines the individual results obtained by each detector [13][17]. Besides, evidence theory has been introduced to edge detection in color images to increase the edge positioning precision from color image results, e.g., a edge detection method in color images based on Dezert-Smarandache Theory (DSmT) [19] was proposed in [18]. It combines the results obtained by grayscale edge detectors for individual color channels with DSmT. Vector methods treat the color information as color vectors in a vector space provided with a vector norm, including gray image edge detector vectorization method [20], multidimensional gradient method [21], vector order statistics method [22], etc.

The color image edge detectors based on multichannel information fusion usually use fusion approaches to combine the individual results obtained from traditional gray edge detection method in each channel to obtain edge in color space [23], such as RGB, HSI and HSV. However, the edge detection results in three channels sometimes are conflicting and discordant and edge detection in single channel based on traditional gray image edge detection generates uncertainty. How to fuse them rationally to obtain the final edge is a challenging task. To obtain the final edge according to the edge information in three channels can be considered as a Multi-Criteria decision making (MCDM) problem. As cautious ordered weighted averaging(OWA) with evidential reasoning (COWA-ER) [24] and Fuzzy-Cautious OWA with evidential reasoning (FCOWAER) [25] are effective approaches to make decision in the framework of MCDM. In this paper, we propose a new edge detector for color images based on COWA-ER and FCOWAER to deal with the uncertainty and discordance. We present experiments of our new edge detection method on two color images and their noisy version. The comparison between our proposed approaches and traditional Sobel detector, Kirsch detector based on gray conversion, edge detector based on Dempster's rule [18] are provided. Experimental results show the effectiveness and rationality of our new edge detector.

\section{TRADITIONAL COLOR IMAGE EDGE DETECTOR BASED ON GRAY CONVERSION}

The edge widely used can be divided into two kinds [26]. One is the step-edge, the other is the roof-edge, as shown 
in Fig.1. The gray value of the pixels on the two sides of the step-edge has a step change. The roof-edge refers to gray value of the pixel gradually increasing to a certain extent and then slowly decreasing. Based on such properties, the image edge can be determined by the derivative of its pixel gray value from the mathematical standpoint.

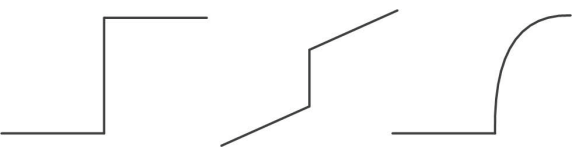

(a) step-edge

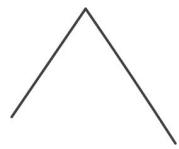

(b) roof-edge
Fig. 1. Edge types.

Traditional color image edge detectors based on gray conversion first convert the color image to gray image and then use traditional gray image edge detectors to detect edge. Traditional gray image edge detection uses gradient maximum or zero-crossing based on gray discontinuity on edge. This method first uses edge detection operator to calculate the gradient of each pixel in the image. Then a threshold is set to detect the edge. If the gradient value is greater than the threshold, the pixel point is detected as belonging to an edge. We briefly recall the two main edge detection operators encountered in the applications: 1) Sobel operator [7], and 2) Kirsch operator [8].

\section{A. Sobel operator}

The Sobel operator is a commonly used first order differential operator. It uses two Sobel kernels to calculate the gradient approximation including the gradient orientation and magnitude which are presented as follows:

$$
G_{x}=\left[\begin{array}{lll}
-1 & 0 & 1 \\
-2 & 0 & 2 \\
-1 & 0 & 1
\end{array}\right] * A \quad G_{y}=\left[\begin{array}{ccc}
-1 & -2 & -1 \\
0 & 0 & 0 \\
1 & 2 & 1
\end{array}\right] * A
$$

where $G_{x}$ and $G_{y}$ are vertical and horizontal gradient components. $A$ is the original image. $*$ denotes the 2-dimensional convolution operation.

Therefore, gradient magnitude is given by:

$$
G_{\text {Sobel }}=\sqrt{G_{x}^{2}+G_{y}^{2}}
$$

\section{B. Kirsch operator}

The Kirsch operator consists of eight convolutions kernels (template). Each pixel in the image is convolved with the eight kernels. The maximum value is the output result of Kirsch operator.

From the eight directions templates shown in Fig.2, it can be seen that the included angle between every two convolutions kernels (templates) is $45^{\circ}$. For an image $A$, the template is $W_{k}(k=1,2, \ldots, 8)$, and the gradient magnitude is:

$$
G_{\text {Kirsch }}=\max \left\{W_{k} * A\right\}
$$

\begin{tabular}{|c|c|c|c|c|c|c|c|c|c|c|c|}
\hline 5 & 3 & 3 & 3 & 3 & 3 & 3 & 3 & 3 & 3 & 5 & 3 \\
\hline-5 & 0 & 3 & -5 & 0 & 3 & 3 & 0 & 3 & 3 & 0 & -5 \\
\hline-5 & 3 & 3 & -5 & -5 & 3 & -5 & -5 & -5 & 3 & -5 & -5 \\
\hline 3 & 3 & -5 & 3 & -5 & -5 & -5 & -5 & -5 & -5 & -5 & 3 \\
\hline 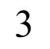 & 0 & -5 & 3 & 0 & -5 & $J$ & 0 & 3 & -5 & 0 & 3 \\
\hline 3 & 3 & -5 & 3 & 3 & 3 & 3 & 3 & 3 & 3 & 3 & \\
\hline
\end{tabular}

The Sobel operator is simple and has a small calculation cost, but it only considers horizontal and vertical templates,
Fig. 2. Kirsch Operator template.

which lose the non vertical and non horizontal possible parts of edge information. Kirsch operator uses 8-direction templates, which can produce the largest gradient boundary. So, the Kirsch operator can extract more details of the edge. However, it may retain more interference noise.

Edge detection methods based on operators usually detect the edge position by the gradient and the detection of edge position is based on single threshold. However, there exists uncertainty near the single threshold which may lead to false alarms under noise. To reduce the effect of noise on edge detection results, we use double thresholds to describe the uncertainty of the gradient interval. Then, we use MCDM to deal with the uncertain information.

\section{NOVEL EDGE DETECTION METHOD BASED ON MCDM WITH EVIDENTIAL REASONING}

In different color spaces, we can divide color images into three channels and then use traditional gray image edge detectors to obtain the edge in each channel. The final edge images are obtained through a fusion step according to chosen rules, as shown in Fig.3. To address discordant results of edge detection in three channels and uncertainty during combining results, we introduce COWA-ER and FCOWA-ER approaches to solve this MCDM problem.

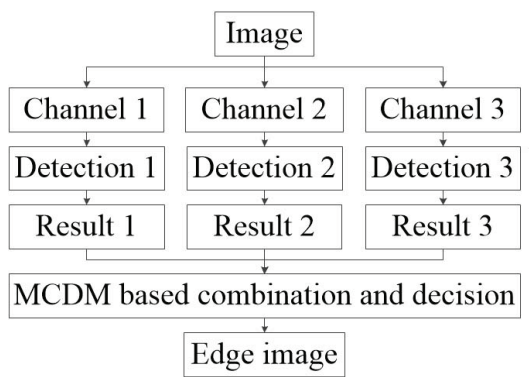

Fig. 3. The block diagram of MCDM.

\section{A. Basics of COWA-ER and FCOWA-ER}

Cautious OWA with evidential reasoning (COWA-ER) [24] and Fuzzy-Cautious OWA with evidential reasoning (FCOWAER) [25] are uncertainty reasoning approaches under the framework of Multi-Criteria decision making (MCDM). 
Let consider the following matrix $C$ provided to a decision maker:

$$
C=\begin{gathered}
S_{1} \\
A_{1} \\
\vdots \\
A_{u} \\
\vdots \\
A_{q}
\end{gathered} \quad\left[\begin{array}{ccccc}
C_{11} & \cdots & C_{1 v} & \cdots & C_{1 r} \\
\vdots & & \vdots & & \vdots \\
C_{u 1} & \cdots & C_{u v} & \cdots & C_{u r} \\
\vdots & & \vdots & & \vdots \\
C_{q 1} & \cdots & C_{q v} & \cdots & C_{q r}
\end{array}\right]
$$

where $A_{u}(u=1, \cdots, q)$ corresponds to a possible alternative available to the decision maker. $S_{v}(v=1, \cdots, r)$ corresponds to a possible value of the information source. $C_{u v}$ is a possible value corresponding the alternative $A_{u}$ and its information source $S_{v}$.

Then the pessimistic and optimistic OWA are used respectively to construct the intervals of expected payoffs for different alternatives. If there exist $q$ alternatives, the expected payoff vector is as follows.

$$
E(C)=\left[\begin{array}{c}
E\left(C_{1}\right) \\
E\left(C_{2}\right) \\
\vdots \\
E\left(C_{k}\right)
\end{array}\right]=\left[\begin{array}{c}
{\left[C_{1}^{\min }, C_{1}^{\max }\right.} \\
{\left[C_{2}^{\min }, C_{2}^{\max }\right]} \\
\vdots \\
{\left[C_{q}^{\min }, C_{q}^{\max }\right]}
\end{array}\right]
$$

\section{1) Principle of COWA-ER:}

Step 1: Divide each bound of intervals by the max of the bounds to obtain a new normalized imprecise expected payoff vector $E^{\operatorname{Imp}}(C)$.

Step 2: Consider the finite set of alternatives $\Theta=\left\{A_{1}, A_{2}, \cdots, A_{q}\right\}$ as the frame of discernment and the sources of belief associated with them are obtained from the normalized imprecise expected payoff vector $E^{\operatorname{Imp}}(C)$. The modeling for computing Basic Belief Assignment ( BBA) associated to $A_{u}$ from any imprecise value $[a ; b] \subseteq[0 ; 1]$ is simple and is done as follows:

$$
\left\{\begin{array}{l}
m_{u}\left(A_{u}\right)=a \\
m_{u}\left(\bar{A}_{u}\right)=1-b \\
m_{u}\left(A_{u} \cup \bar{A}_{u}\right)=b-a
\end{array}\right.
$$

where $\bar{A}_{u}$ is the $A_{u}$ 's complement in $\Theta$.

Step 3: Use Dempster's rule to combine $m_{u}(\cdot)$.

Dempster's rule of combination [27] is defined as

$$
m(A)=\frac{\sum_{B \cap C=A} m_{1}(B) \cdot m_{2}(C)}{1-K} \quad A \neq \emptyset
$$

where $K=\sum_{B \cap C=\emptyset} m_{1}(B) \cdot m_{2}(C)$ denotes the conflicting coefficient.

Step 4: Obtain the decision result based on the pignistic probabilities obtained by using the pignistic transformation.

Pignistic probability transformation [28] is a transformation from a BBA into a probability for probabilistic decisionmaking:

$$
\operatorname{BetP}\left(A_{u}\right)=\sum_{A_{u} \in A_{M}, A_{M} \subseteq 2^{\ominus}} \frac{m\left(A_{M}\right)}{\left|A_{M}\right|}
$$

where $\left|A_{M}\right|$ is the cardinality of $A_{M}$.

\section{2) Principle of FCOWA-ER:}

Step 1: Consider the two columns of $E(C)$ as two information sources, representing the pessimistic and the optimistic attitudes. The column-wise normalized expected payoff is

$$
E^{\text {Fuzzy }}(C)=\left[\begin{array}{c}
N_{1}^{\min }, N_{1}^{\max } \\
N_{2}^{\min }, N_{2}^{\max } \\
\vdots \\
N_{q}^{\min }, N_{q}^{\max }
\end{array}\right]
$$

where the vectors $\left[N_{1}^{\min }, N_{2}^{\min }, \cdots, N_{q}^{\min }\right]$ and $\left[N_{1}^{\max }\right.$, $\left.N_{2}^{\max }, \cdots, N_{q}^{\max }\right]$ can be seen as two FMFs representing pessimistic and optimistic attitudes.

Step 2: Convert two FMF into two BBAs $m_{P e s s}$ and $m_{O p t i}$ by using $\alpha$-cut approach [29]. Suppose the frame of discernment (FOD) is $\Theta=\left\{A_{1}, A_{2}, \cdots, A_{q}\right\}$ and the Fuzzy Membership Functions (FMFs) is $\mu\left(A_{u}\right)(u=1,2, \cdots, k)$. The corresponding BBA introduced is used to generate $M$ $\alpha$-cut $\left(0<\alpha_{1}<\alpha_{2}<\cdots<\alpha_{M} \leq 1\right)$, where $M \leq|\Theta|=$ $n$.

$$
\begin{gathered}
B_{v}=\left\{A_{u} \in \Theta \mid \mu\left(A_{u}\right) \geq \alpha_{u}\right\} \\
m\left(B_{v}\right)=\frac{\alpha_{v}-\alpha_{v-1}}{\alpha_{M}}
\end{gathered}
$$

where $B_{v}(v=1,2, \cdots, M)$ is the focal element. Here we set $M=k$ and $0<\alpha_{1}<\alpha_{2}<\cdots<\alpha_{q} \leq 1$ as the sort of $\mu\left(A_{u}\right)$.

Step 3: Use Dempster's rule to combine $m_{P e s s}(\cdot)$ and $m_{O p t i}(\cdot)$.

Step 4: Obtain the decision result based on the max of the pignistic probabilities obtained by using the pignistic transformation.

\section{B. Edge detection based on COWA-ER and FCOWA-ER}

In this paper, we process color images in RGB (Red, Green, Blue) color space. RGB color space specifies the color using three components: R, G, B. Let us consider the three components as three color channels (layers). The frame of discernment (FOD) $\Theta$ for edge detection is defined as

$$
\Theta=\left\{\theta_{1} \triangleq \text { Pixel } \in \text { Edge, } \theta_{2} \triangleq \text { Pixel } \notin \text { Edge }\right\}
$$

For color image edge detection such a Multi-Criteria decision problem, a decision matrix $C$ is defined as follows:

$$
\theta_{1} \quad\left[\begin{array}{ccc}
R & G & B \\
\theta_{2}^{R} & C_{1}^{G} & C_{1}^{B} \\
C_{2}^{R} & C_{2}^{G} & C_{2}^{B}
\end{array}\right]=C
$$

where $C_{1}^{L}$ and $C_{2}^{L}$ are the evaluations that each pixel for each color layer belong to the edge and non-edge. $L$ is denoted as $\mathrm{R}, \mathrm{G}, \mathrm{B}$.

As in traditional gray image edge detection method, an operator is used to calculate the gradient at first. Suppose the size of the image in each layer is $M \times N$. For each pixel point $(i, j)(i=1,2, \cdots, M, j=1,2, \cdots, N)$, we calculate the gradient using the Sobel operator as follows:

$$
g^{L}(i, j)=\left(G_{x}^{L}(i, j)^{2}+G_{y}^{L}(i, j)^{2}\right)^{1 / 2}
$$


where

$$
\left\{\begin{array}{l}
G_{x}^{L}=W_{x} * A^{L} \\
G_{y}^{L}=W_{y} * A^{L}
\end{array}\right.
$$

We can also calculate the gradient by using the Kirsch operator:

$$
g^{L}(i, j)=\max \left\{G_{1}, G_{2}, G_{3}, G_{4}, G_{5}, G_{6}, G_{7}, G_{8}\right\}
$$

where

$$
G_{k}=W_{k} * A^{L}(k=1,2, \cdots, 8)
$$

It is worth noting that the range of gradient magnitude $\left[g_{\min }^{L}, g_{\max }^{L}\right]$ in each layer is not of the same order. So, we need to carry out Min-Max Normalization before the next step, which is a linear transformation of the original gradient data. The result value is mapped to $[0,255]$. The transfer function is as follows:

$$
g_{n}^{L}(i, j)=\frac{g^{L}(i, j)-g_{\min }^{L}}{g_{\max }^{L}-g_{\min }^{L}} \cdot 255
$$

where $g_{n}^{L}(i, j)$ is gradient value mapped to $[0,255]$ in each layer. $g_{\max }^{L}$ and $g_{\min }^{L}$ are the maximum and minimum values in the gradient values of the entire image in each layer respectively.

Traditional gray image edge detections usually make decision thanks to a single threshold. If the gradient value is below the threshold, then this pixel is judged as the nonedge point, otherwise it is judged as edge point. However, this direct judgment is sensitive to noise and there also exists uncertainty, and it will lead to losing edge information and generating false alarms. Therefore, we use double thresholds to describe the uncertainty and implement the approach based on MCDM with evidential reasoning in edge detection to address the uncertainty.

We select double thresholds $T^{L}=\left[t_{l}^{L}, t_{h}^{L}\right]$ based on gradient histogram. In the gradient image, the grayscale smoothing area of the main body in the original image has smaller value and the edge area has larger value. As illustrated in Fig.4 for Lena's image, most pixels are distribute in the part of smaller gradient. As a statistics, the mean reflects the main feature, and the standard deviation reflects the deviation of the sample from the subject [21]. Combined with gradient mean and standard deviation method one can select the local threshold to achieve better results. These thresholds are defined by

$$
\left\{\begin{array}{c}
t_{l}^{L}=\mu^{L}+k_{l} \delta^{L} \\
t_{h}^{L}=\mu^{L}+k_{h} \delta^{L}
\end{array}\right.
$$

where

$$
\begin{gathered}
\mu^{L}=\frac{\sum_{i=1}^{i=M} \sum_{j=1}^{j=N} g_{n}^{L}(i, j)}{M \cdot N} \\
\delta^{L}=\sqrt{\frac{\sum_{i=1}^{i=M} \sum_{j=1}^{j=N}\left[g_{n}^{L}(i, j)-\mu^{L}\right]^{2}}{M \cdot N}}
\end{gathered}
$$

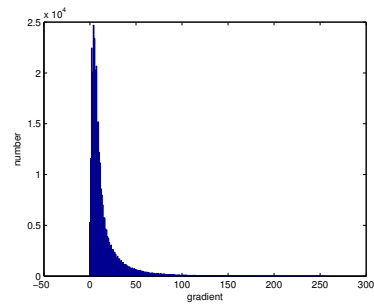

(a) Original Lena image

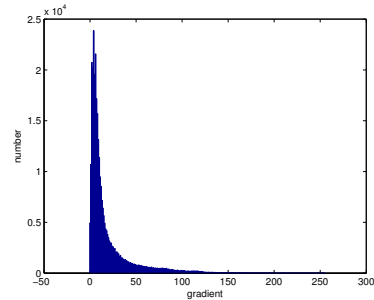

(b) Lena image with noise
Fig. 4. Gradient histogram of Lena image based on Sobel operator.

and where $t_{l}$ is the low threshold and $t_{h}$ is the high threshold in each layer. $\mu$ is the gradient mean and $\delta$ is the gradient standard deviation. $k_{l}$ and $k_{h}$ are proportionality coefficients.

It will provide more edge details when $k_{h}$ is assigned smaller value. Instead, it will provide the main edges of the image when $k_{l}$ is assigned larger value. The mean value of gradient magnitude indicates the main feature of the image, which is no-edge area in the image. Therefore, low threshold $\left(t_{l}\right)$ should be assigned near the mean and $k_{l}$ can be taken near 0 . The standard deviation of gradient magnitude can be understood as the discrete degree of distance from main feature ( non-edge area). So we can take the value of $k_{h}$ around 1.0 under low noise conditions. With the increasing level of noise, which means more uncertainty, the interval length of $\left[t_{l}, t_{h}\right]$ should be taken larger, which requires to decrease $k_{l}$ and increase $k_{h}$.

In edge detection, if the gradient value of measured pixel is close to the threshold, it means a greater greater uncertainty. Instead, far from the threshold means the larger reliability of the pixel belonging to edge or non-edge. According to this feature, let us consider the hyperbolic tangent function defined as

$$
f_{\lambda, t}(g) \triangleq \frac{\tanh [-\lambda(g-t)]+1}{2}
$$

where $g$ is the gradient magnitude of the pixel. $t$ is the threshold for edge detection segmentation. $\lambda$ is the slope coefficient as an empirical constant. The mutative curves of functions $f_{\lambda, t_{h}}(g)$ and $f_{-\lambda, t_{l}}(g)$ which represent the evaluations of edge and non-edge respectively are shown in Fig.5.

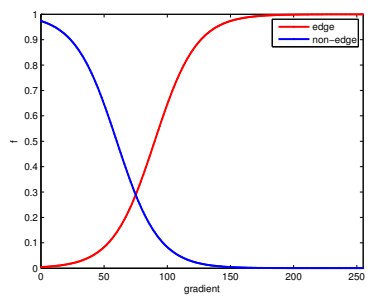

(a)

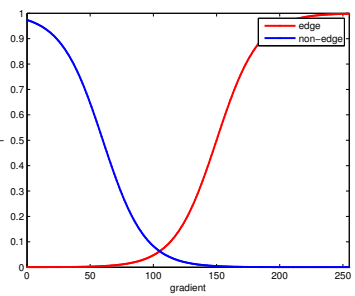

(b)
Fig. 5. Functional relationship graphics $(\lambda=0.03)$ (a) $\left[t_{l}, t_{h}\right]=[60,90]$ (b) $\left[t_{l}, t_{h}\right]=[60,150]$. 
In this paper, the Sobel operator and Kirsch operator are respectively used to calculate gradient magnitude to generate the decision matrix. The following decision matrix $C(i, j)$ for each pixel point can be constructed as

$$
\left[\begin{array}{ccc}
f_{\lambda, t_{h}^{R}}\left(g_{n}^{R}(i, j)\right) & f_{\lambda, t_{h}^{G}}\left(g_{n}^{G}(i, j)\right) & f_{\lambda, t_{h}^{B}}\left(g_{n}^{B}(i, j)\right) \\
f_{-\lambda, t_{l}^{R}}\left(g_{n}^{R}(i, j)\right) & f_{-\lambda, t_{l}^{G}}\left(g_{n}^{G}(i, j)\right) & f_{-\lambda, t_{l}^{B}}\left(g_{n}^{B}(i, j)\right)
\end{array}\right] \text { (19) }
$$

where $g_{n}^{L}(i, j)$ is the normalized gradient magnitude of the pixel to be detected.

Then the pessimistic and optimistic OWA used respectively to construct the intervals of expected payoffs for edge and non-edge are as follows.

$$
E(C(i, j))=\left[\begin{array}{c}
{\left[f_{\lambda, t_{h}^{L}}^{\min }\left(g_{n}^{L}(i, j)\right), f_{\lambda, t_{h}^{L}}^{\max }\left(g_{n}^{L}(i, j)\right)\right]} \\
{\left[f_{-\lambda, t_{l}^{L}}^{\min }\left(g_{n}^{L}(i, j)\right), f_{-\lambda, t_{l}^{L}}^{\max }\left(g_{n}^{L}(i, j)\right)\right]}
\end{array}\right]
$$

In the final we make decision by using COWA-ER or FCOWA-ER methods aforementioned and obtain pignistic probability based on the combined BBAs. The detailed implementation of COWA-ER and FCOWA-ER are as described below.

\section{1) Implementation of COWA-ER:}

Step 1: Divide each bound of intervals in $E(C(i, j))$ by the max of the bounds to obtain a new normalized imprecise expected payoff vector $E^{\operatorname{Imp}}(C(i, j))$.

Step 2: Convert each normalized imprecise value into its BBA according to the transformation (Eq. 5).

Step 3: Use Dempster's rule of combination to obtain the combined BBA $m_{i j}(\cdot)$.

Step 4: Obtain the BBAs corresponding pignistic probability $B e t P_{i j}(\cdot)$ to make the final decision.

2) Implementation of FCOWA-ER:

Step 1: Normalize each column in $E(C(i, j))$ to get $E^{\text {Fuzzy }}(C(i, j))$.

Step 2: Obtain two FMFs and convert them into two BBAs by using $\alpha$-cut approach (Eq. 9)

Step 3: Use Dempster's rule of combination to obtain the combined BBA $m_{i j}(\cdot)$.

Step 4: Obtain the BBAs corresponding pignistic probability $\operatorname{Bet} P_{i j}(\cdot)$ to make the final decision.

In step 4, due to the binary FOD $\Theta$, the pignistic probability for each pixel can be obtained as

$$
\left\{\begin{array}{l}
\operatorname{Bet} P\left(\theta_{1}\right)=m\left(\theta_{1}\right)+\frac{1}{2} m\left(\theta_{1} \cup \theta_{2}\right) \\
\operatorname{BetP}\left(\theta_{2}\right)=m\left(\theta_{2}\right)+\frac{1}{2} m\left(\theta_{1} \cup \theta_{2}\right)
\end{array}\right.
$$

It is easy to find that the final decision making under this frame is the one that has largest pignistic probability. For experiments of edge detection in this paper, The condition $\operatorname{Bet} P\left(\theta_{1}\right)>\varepsilon$ should be added in the process of decisionmaking in order to reduce misjudgment in uncertain situations.

The implementation of method proposed can be summarized as illustrated in Fig.6.

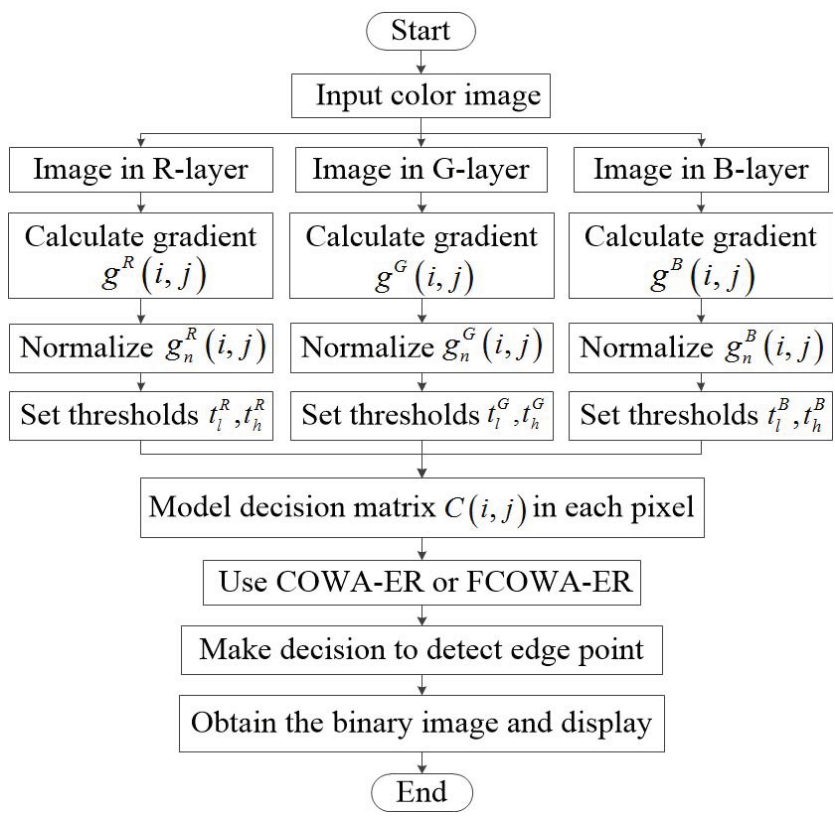

Fig. 6. The block diagram of novel edge detection method

\section{EXPERIMENTS AND ANALYSIS}

In the previous section, we introduced two traditional color image edge detection methods based on gray conversion: Sobel edge detector (hereinafter referred to as Sobel) and Kirsch edge detector (Kirsch). We mentioned edge detector based on Dempster's rule (S) [18]. Beside, we proposed new edge detectors for color based on MCDM with evidential reasoning, including edge detector based on COWA-ER with Sobel operator (COWA(S)), edge detector based on FCOWAER with Sobel operator (FCOWA(S)), edge detector based on COWA-ER with Kirsch operator $(\mathrm{COWA}(\mathrm{K}))$, edge detector based on FCOWA-ER with Kirsch operator (FCOWA(K)).

Lena image is the most widely used reference image in the edge detection, chessboard image has rich and variational color with clear edges, and ice candy image has rich edge. So we experiment based on described edge detectors and our proposed edge detectors with the three color images. The original Lena image, chessboard image and ice candy image are shown on Fig.7(a), Fig.8(a) and Fig.13(a), and the corresponding grayscales are shown on Fig.7(b), Fig.8(b) and Fig.13(b). Then we make comparisons to analyze methods. For better comparisons among different methods, we test these edge detection methods under both noise-free and noisy conditions.

\section{A. Original image}

In this simulation, we take $\lambda=0.03$. We test the methods on Lena image by setting $k_{l}=0.4, k_{h}=1.6$ and test on chessboard image by setting $k_{l}=0.1, k_{h}=0.8$. The results of different edge detectors on original Lena image and chessboard image are shown in Fig.7 and Fig.8 respectively.

As shown in Fig.7, our proposed methods and other edge detectors have similar detection performance when tested on 

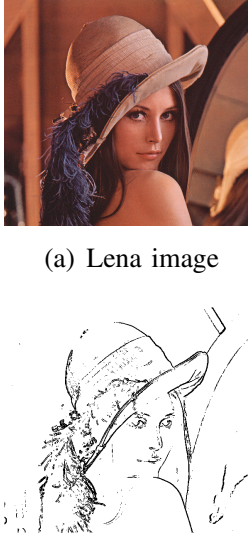

(d) Kirsch

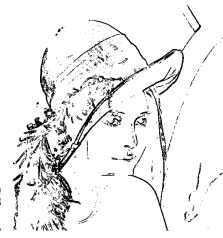

(g) $\operatorname{COWA}(\mathrm{K})$ (a) Lena image

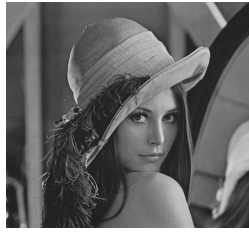

(b) Grayscale

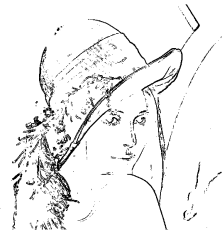

(e) DS

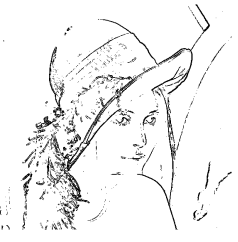

(c) Sobel
Fig. 7. Comparative edge detection results of Lena image.

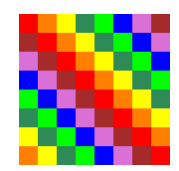

(a) original

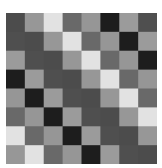

(b) Grayscale

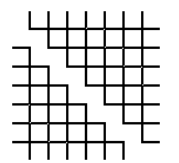

(c) Sobel

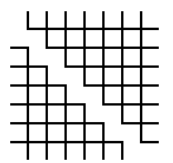

(d) Kirsch

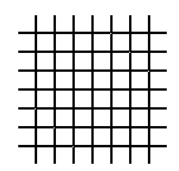

(e) $\mathrm{DS}$

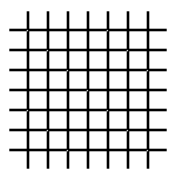

(f) $\operatorname{COWA}(\mathrm{S})$

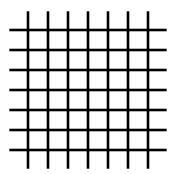

(g) $\operatorname{COWA}(\mathrm{K})$

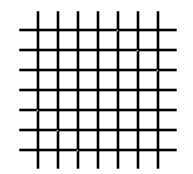

(h) $\operatorname{FCOWA}(\mathrm{S})$

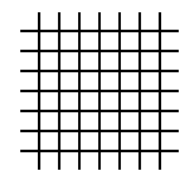

(i) $\operatorname{FCOWA}(\mathrm{K})$
Fig. 8. Comparative edge detection results of chessboard image.

Lena image. However, as in Fig.8, our edge detector and DS detector is much better than Sobel and Kirsch edge detectors when tested on colourful chessboard image. Because traditional color image edge detectors need to convert the color image to gray image Fig.8(b). By doing this, we loose a part of the image information during conversion so that Sobel and Kirsch edge detectors can not provide a complete edge. The edge detection methods based on fusion make full use of the color information and give a complete edge which overcome the shortcomings of traditional color image edge detector in this area.

\section{B. Noisy image}

In this simulation, we take $\lambda=0.08$. We add salt and pepper with different noise densities (den) to Lena image and ice candy image in each color layer. In blue layer, we add den $=5 \%$ salt and pepper noise and den $=1.25 \%$ noise in other layers. The Sobel edge detection results in each color layers are shown in Fig.9 and Fig.11, which are very noisy and have more false alarms. We test the new edge detection methods on Lena image by setting $k_{l}=0.2, k_{h}=2.8$ and on chessboard image by setting $k_{l}=-0.1, k_{h}=1.1$. The results of different edge detectors on original Lena image and chessboard image are shown in Fig.10, and Fig.12 respectively. Besides, we also did make a test on ice candy image of Fig.13 which has rich edge in order to show the difference between the detection results under noise. We increase the noise level in adding den $=10 \%$ salt and pepper noise in blue layer and den $=2.5 \%$ noise in other layers. Here we set $k_{l}=0.1, k_{h}=1.8$. The results of different edge detectors on original ice candy image are shown in Fig.14.

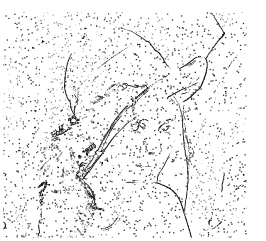

(a) R-layer

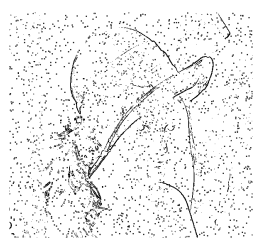

(b) G-layer

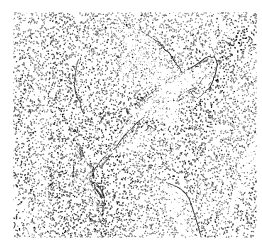

(c) B-layer
Fig. 9. Sobel detector in each layer on noisy Lena image.

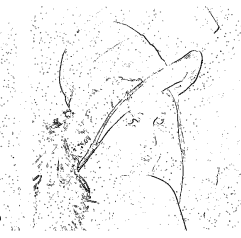

(a) Sobel

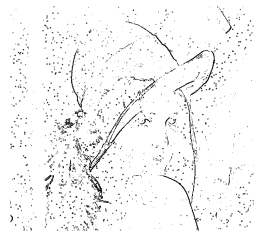

(b) Kirsch

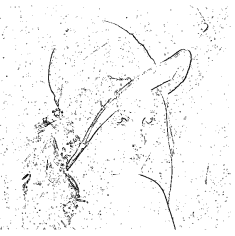

(c) DS

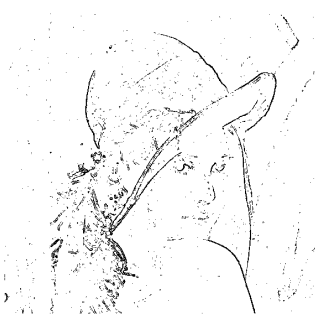

(d) $\operatorname{COWA}(\mathrm{S})$

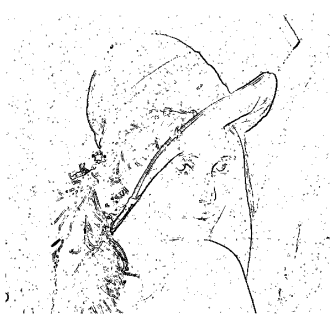

(f) $\operatorname{FCOWA}(\mathrm{S})$

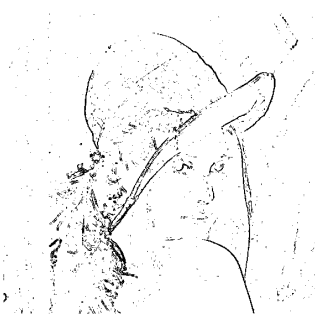

(e) $\operatorname{COWA}(\mathrm{K})$

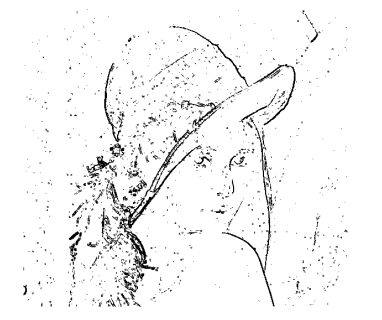

(g) $\mathrm{FCOWA}(\mathrm{K})$

Fig. 10. Comparative edge detection results of Lena image with noise.

The comparisons between the results of different edge detection methods shown in Fig.10, Fig.12 and Fig.14 indicate that our proposed methods give a better detection performance 


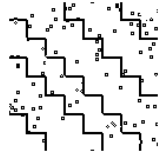

(a) R-layer

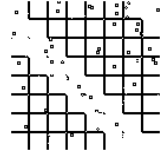

(b) G-layer

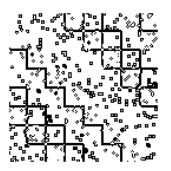

(c) B-layer
Fig. 11. Sobel detector in each layer on noisy chessboard image.

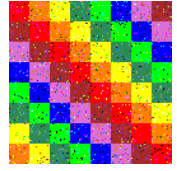

(a) with noise

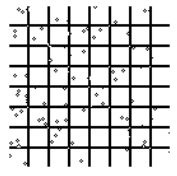

(e) $\operatorname{COWA}(\mathrm{S})$

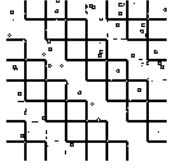

(b) Sobel

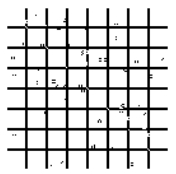

(f) $\operatorname{COWA}(\mathrm{K})$

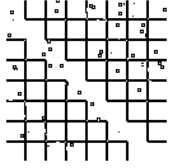

(c) Kirsch

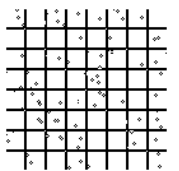

(g) $\operatorname{FCOWA}(\mathrm{S})$

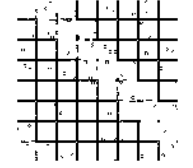

(d) DS

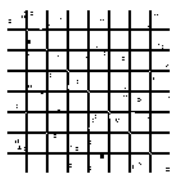

(h) $\mathrm{FCOWA}(\mathrm{K})$
Fig. 12. Comparative edge detection results of chessboard image with noise.

under noisy image. As shown in Fig.10(d) - 10(g), Fig.12(e) 12(h) and Fig.14(e) - 14(h), the results show that the edge detection based on evidence reasoning method can detect weak edge and have the capability of noise immunity. It is superior to the traditional color image edge detectors based on gray conversion. Compared with DS edge detector shown by Fig.10(c), Fig.12(d) and Fig.14(d), our experimental result indicates that the new edge detection approaches achieve better image processing effect, because they have a stronger ability of eliminating noise as well as keeping clear image edge.

\section{Quantitative evalation with Pratt's figure of merit}

Pratt's figure of merit [30] is a quantitative index proposed by Pratt for the objective evaluation of the performance of edge detection operators. It is also a more comprehensive quantitative evaluation parameter, which is used as an objective evaluation criterion in this paper. Pratt's figure of merit mainly examines three kinds of detection errors: (a) loss of effective edge, (b) edge positioning error, (c) the noise to determine the edge. The figure of merit is defined as

$$
F=\frac{1}{\max \left(I_{A}, I_{I}\right)} \sum_{i=1}^{I_{A}} \frac{1}{1+a d_{i}^{2}}
$$

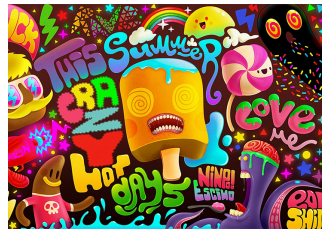

(a) original image

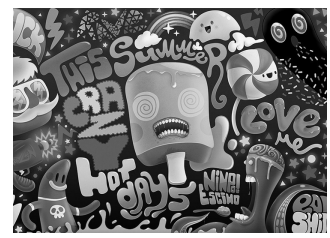

(b) grayscale
Fig. 13. Ice candy image.

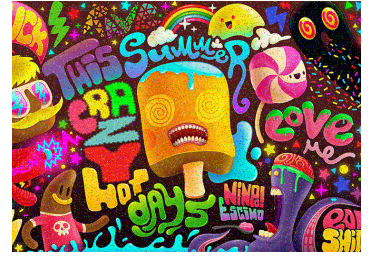

(a) ice candy image with noise

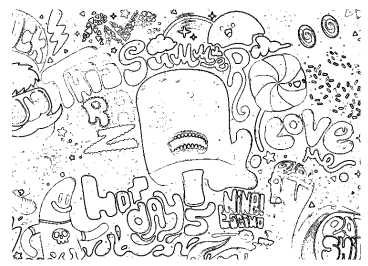

(c) Kirsch

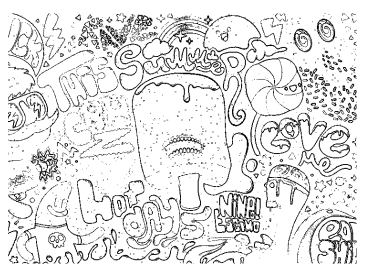

(e) $\operatorname{COWA}(\mathrm{S})$

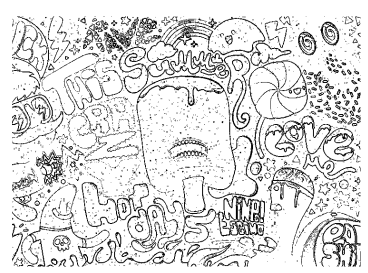

(g) $\operatorname{FCOWA}(\mathrm{S})$

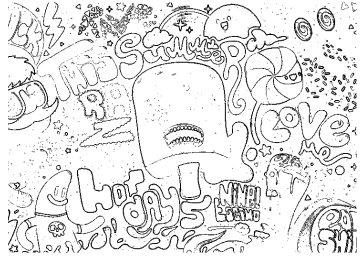

(b) Sobel

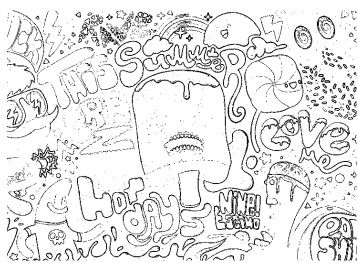

(d) DS

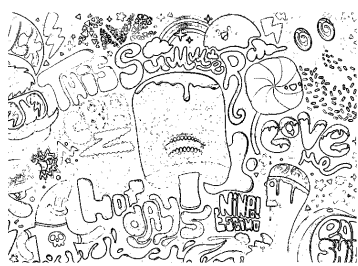

(f) $\operatorname{COWA}(\mathrm{K})$

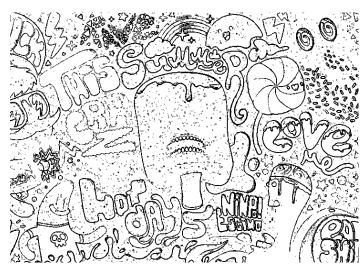

(h) $\operatorname{FCOWA}(\mathrm{K})$
Fig. 14. Comparative edge detection results of ice candy image with noise.

where $I_{A}$ and $I_{I}$ are the number of actual and ideal edge points. $d_{i}$ is the pixel miss distance of the $i$ th edge detected, and $a$ is a scaling constant. The value of Pratt's figure of merit is a positive number less than or equal to 1 . The greater value of Pratt's figure of merit means the better performance of the detector.

We plot the Pratt's figure of merit as a function of signal-tonoise ratios (SNR) for different detectors on color chessboard image as shown in Fig.15. As the curve variation tendencies shown, the performance of edge detection based on COWAER and FCOWA-ER are approximate to each other. Due to the loss of color information in the process of converting to grayscale image, the Pratt's figure of merit value of Sobel and Kirsch detector can not exceed 0.9. Our new proposed method has better performances compared with traditional color image edge detection methods based on gray conversion at a high SNR, which just confirms the above experiment. When there is a low SNR, the Pratt's figure of merit value of our proposed detector is higher than that of DS edge detector, which indicates the edge detection based on COWA-ER and FCOWA-ER can distinguish noise points effectively. 


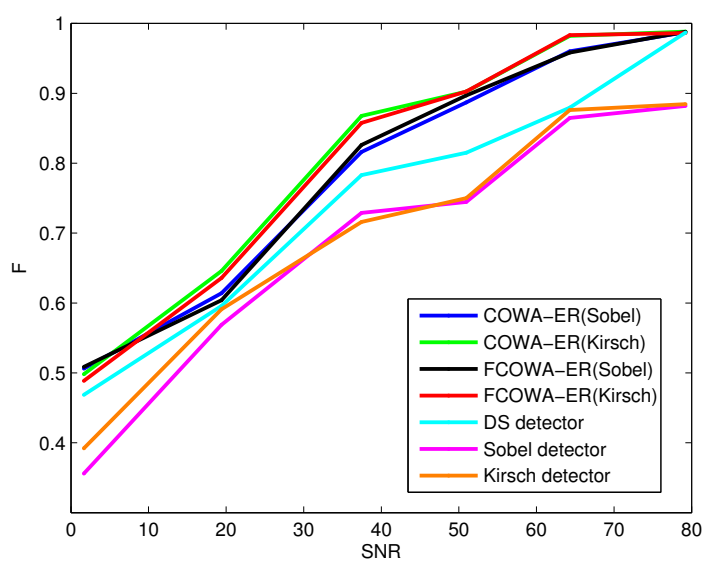

Fig. 15. Figure of merit comparison on chessboard image.

\section{Conclusions}

In this paper, we have presented novel edge detection methods for color image based on MCDM with evidential reasoning and the commonly used edge detection operators. We did model decision matrix for each pixel in the RGB image according to its gradient magnitude in each layer calculated by using Sobel or Kirsch operator. A double thresholding mechanism based on gradient histogram has been used to address the uncertain information during edge detection. Then, COWA-ER and FCOWA-ER were introduced to obtain the final decision. In our experiments, our new proposed methods have shown better performances and robustness with respect to other methods, specially on noisy images. These new edge detectors can not only overcome the uncertainty of edge to some extent and extract more details of edge, but also effectively eliminate the impact of noise.

In the future work, we would like to improve these edge detection approaches. We will apply some other combination rules in COWA-ER or FCOWA-ER to fuse the BBAs and attempt to develop the method of adaptive double thresholding. Furthermore, we will try to generalize our idea in other color space like HSV (Hue, Saturation, Value) color space.

\section{ACKNOWLEDGMENT}

This work was supported by the National Natural Science Foundation (Nos. 61573275, 61671370), Grant for State Key Program for Basic Research of China (973) (No. 2013CB329405), Science and Technology Project of Shaanxi Province (No. 2013KJXX-46), Postdoctoral Science Foundation of China (No. 2016M592790), and Fundamental Research Funds for the Central Universities (No. xjj2014122, xjj2016066).

\section{REFERENCES}

[1] P. Arbelaez, M. Maire, et al, Contour detection and hierarchial image segentation, IEEE Trans on PAMI, 2010, 33 (5): 898-916.

[2] A. Spacek, Edge detection and motion detection, Image and vision computing, 1986, 4 (1): 43-56.
[3] Y. Gao, M. K. H. Leung, Face recognition using line edge map, IEEE Trans on PAMI, 2002, 24 (6): 764-779.

[4] J. Zeng, Research on Image Edge Detection and Its APPlication, Ph.D. Thesis, Huazhong University of Science and Techonlogy, China, 2011.

[5] L. G. Roberts, Machine perception of three-dimensional solids, In Optical and Electro-Optical Information Processing, Cambridge, MA: MIT Press, 1965.

[6] J. M. Prewitt, Object enhancement and extraction, New York: Academic Press, 1970.

[7] I. Sobel, Camera models and machine perception, Ph.D. Thesis, Stanford University, Stanford, CA, 1970.

[8] R. A. Kirsch, Computer determination of the constituent structure of biological images, Computer and Biomedical Research, 1971, 4 (3): 315328.

[9] J. Canny, A computational approach to edge detection, IEEE Trans on Pattern Analysis and Machine Intelligence, 1986, 8 (6): 679-698

[10] S. M. Smith, J.M.Brady, SUSAN-A new approach to low level image processing, International Journal of Computer Vision, 1997, 23 (1): 4578.

[11] A. Koschan, M.Abidi, Detection and classification of edges in color images, Signal Processing Magazine, 2005,22 (1): 64-73

[12] T. Yang, M. Gu, et al, Review of color image edge detection algorithms, Application Research of Computers, 2015, 32 (9): 2566-2571.

[13] R. Nevatia, Color edge detector and its use in scene segmentation, IEEE Trans on Systems Man and Cybernetics, 1977, 7 (11): 820-826

[14] M. Hedley, H. Yan, Segmentation of color images using spatial and color space information, Journal of Electronic Imaging, 1992, 1(4): 374-380

[15] T. Carron, P. Lambert, Color edge detection using jointly huesaturation and intensity, Proc of IEEE International Conference on Image Processing, 1994: 977-981

[16] X. Chen, H. Chen, A novel color edge detection algorithm in RGB color space, Proc of the 10th International Conference on Signal Processing, 2010: 793-796

[17] M. D. Arpitha, M. P. Arakeri, G. R. M. Reddy, An approach for color edge detection with automatic threshold detection, Advanced ComputingNetworking and Security, Berlin: Springer, 2012: 117-124

[18] J. Dezert, Z. Liu, G. Mercier, Edge Detection in Color Images Based on DSmT, 14th International Conference on Information Fusion, Chicago USA, July 5-8, 2011.

[19] F. Smarandache, J. Dezert, Advances and Applications of DSmT for Information Fusion, American Research Press, Rehoboth, Vol.1-3, 20042009.

[20] J. Zeng, D. Li, SUSAN edge detection method for color image, Computer Engineering and Applications, 2011, 47(15)194-196.

[21] R. K. Sidhu, Improved canny edge detector in various color spaces, International Conference on Reliability, INFOCOM Technologies and Optimization, IEEE, 2014: 1-6.

[22] R. Lukac, B. Smolka, et al, Vector filtering for color imaging, IEEE Signal Processing Magazine, 2005, 22 (1): 74-86.

[23] E. Dubois, The structure and properties of color spaces and the representation of color images, Synthesis Lectures on Image,Video,and Multimedia Processing.Morgan and Claypool, 2009.

[24] J. M. Tacnet, J. Dezert, Cautious OWA and evidential reasoning for decision making under uncertainty, Proc.of 14th International Conference on Information Fusion Chicago, Illinois, USA, July 5-8, 2011, $2074 C 2081$.

[25] D. Han, J. Dezert, J.-M. Tacnet, C. Han, A Fuzzy-Cautious OWA Approach with Evidential Reasoning, International Conference on Information Fusion.July 9-12, 2012.

[26] R. Liu, Q. Song, C. Wang, Research on Edge Detection Algorithm in Digital Image, Beijing: Science Press, 2015.

[27] G. Shafer, A Mathematical Theory of Evidence, Princeton: Princeton University, 1976.

[28] P. Smets, and R. Kennes, The transferable belief model, Artificial Intelligence, vol.66, no.2, pp. 191-234, 1994

[29] M. C. Florea, A. L. Jousselme, et al, Approximation techniques for the transformation of fuzzysets into random sets, Fuzzy Sets and Systems, 2008, 159 (3): 270-288.

[30] I. E. Abdou, W. K. Pratt, Quantitative Design and Evaluation of Enhancement/Thresholding Edge Detectors, Proc.IEEE, 1979, 67 (5): 753-763. 